\title{
Does passive smoking increase the frequency of health service contacts in children with asthma?
}

\author{
I K Crombie, A Wright, L Irvine, R A Clark, P W Slane
}

\begin{abstract}
Background-Passive smoking is a major cause of respiratory morbidity in children. However, few studies give accurate estimates of the health effects of passive smoking in children with asthma using an objective measure of exposure. The effects of passive smoking using salivary cotinine levels to measure exposure were investigated.
\end{abstract}

Methods-A sample of 438 children aged 2-12 years with asthma who had a parent who smoked were recruited in Tayside and Fife, Scotland. Health service contacts for asthma, assessed from GP case records, were used as a proxy for morbidity.

Results-A weak U-shaped relationship was found between the salivary cotinine level and health service contacts for asthma: compared with low cotinine levels those with moderate cotinine levels had a reduced contact rate (relative rate $(R R)=$ $0.91,95 \%$ confidence interval (CI) 0.80 to 1.05), whereas high cotinine levels were associated with an increased rate of contact $(R R=1.19,95 \%$ CI 1.05 to 1.37$)$. In contrast, a strong association was seen with the amount the parent reported smoking in front of the child: the higher the level the fewer visits were made for asthma ( $R R$ for everyday exposure $=\mathbf{0 . 6 6}$, $95 \%$ CI 0.56 to 0.77 ). This effect was not seen for non-respiratory visits. Demographic factors, age of child, and number of children in the family all had a powerful effect on the number of visits for asthma. The parents' perception of asthma severity was associated with visit frequency independent of actual severity (derived from drug treatment).

Conclusion-High levels of parental smoking in the home are associated with a reduction in health care contacts for asthma. This could be due to a lack of awareness of asthma symptoms among heavy smokers or a reluctance to visit the GP. Children with asthma who have parents who smoke heavily may not be receiving adequate management.

(Thorax 2001;56:9-12)

Keywords: passive smoking; children; asthma; parental smoking; GP visits

Passive smoking is a major cause of respiratory morbidity in children. ${ }^{12}$ There is good evidence that passive smoking increases the prevalence of asthma, ${ }^{3}$ but it is less clear whether passive smoking causes exacerbations of asthma with some studies claiming that it does and others that it does not. ${ }^{4}$ However, these studies often involve small numbers of children with asthma (usually less than 200) and some do not use objective measures of exposure, relying on self-reports by parents. This study investigated whether high levels of passive smoking increased the risk of morbidity in children with asthma using salivary cotinine levels as a measure of exposure. We had previously observed ${ }^{5}$ that many children with asthma were exposed to high levels of passive smoking. This provided the opportunity and the motivation to investigate whether passive smoking affects morbidity. Morbidity was assessed by health service contacts for asthma and for non-respiratory conditions.

\section{Methods}

The study participants had previously taken part in a randomised controlled trial of an intervention to reduce passive smoking by children with asthma. ${ }^{6}$ In 73 general practices in Tayside and Fife, children were identified who had a diagnosis of asthma in their case notes and who had a parent who smoked. Of the 704 families who were eligible, 501 gave informed consent to take part in the clinical trial. Complete data sets were obtained for 438 children.

The index parents completed a questionnaire on socioeconomic factors, the child's asthma, their smoking, and the child's exposure to tobacco smoke from other sources. Social class was based on occupation of the head of the household. ${ }^{7}$ Children were classified in groups by treatment steps according to the British Thoracic Society (BTS) guidelines. ${ }^{8}$ The 1993 guidelines were used as they were current at the time of recruitment to the study. Saliva samples to measure cotinine, the major metabolite of nicotine, were obtained from the index parent and child. ${ }^{9}$ The method used for collection of samples has been previously described. ${ }^{5}$ Written consent to review GP case records was obtained at baseline. The study was approved by the local medical research ethics committees.

Health service contacts were assessed by a research nurse (AW) who was blind to the cotinine levels of the children. Data were obtained from the GP case notes, computerised prescribing records, and asthma clinic records for a 12 month period prior to the collection of the saliva sample for cotinine measurement. Selection of this period ensured that health service contacts were not influenced by the families' participation in the randomised controlled trial. Information was collected on all contacts recorded in the case records, whether sched- 
uled or patient initiated. Contacts were classified as asthma related if the terms "asthma", "rhonchi", or "wheeze" were mentioned or if asthma medication was increased (for example, higher dose of inhaled steroids or prescription for oral steroids). Non-respiratory contacts were those where there was no mention of asthma terms or of upper or lower respiratory conditions, hay fever, tonsillitis, rhinitis, or otitis media. Exposure to tobacco smoke was categorised by cotinine level as low $(\leqslant 2.0 \mathrm{ng} / \mathrm{ml})$, medium (2.1-4.5 ng/ml), or high (>4.5 ng/ $\mathrm{ml})$. Routine child health surveillance screenings and immunisation visits were excluded as were return visits for acute events.

Table 1 Influence of smoking factors on frequency of asthma contacts: univariate analysis

\begin{tabular}{|c|c|c|c|c|c|c|}
\hline Factor & $n$ & $I R R$ & $95 \% C I$ & Model $\chi^{2}$ & $d f$ & $p$ value \\
\hline \multicolumn{7}{|c|}{ Child cotinine level } \\
\hline Low & 135 & 1.00 & - & & & \\
\hline Medium & 153 & 0.91 & 0.80 to 1.05 & & & \\
\hline \multirow{2}{*}{\multicolumn{7}{|c|}{$\begin{array}{l}\text { Amount smoked in } \\
\text { home by index parent } \\
\text { (cigarettes/day) }\end{array}$}} \\
\hline & & & & & & \\
\hline $0-5$ & 104 & 1.00 & - & & & \\
\hline $6-10$ & 191 & 0.81 & 0.71 to 0.92 & & & \\
\hline $11-15$ & 78 & 0.70 & 0.59 to 0.83 & & & \\
\hline $16-20$ & 50 & 0.74 & 0.61 to 0.91 & & & \\
\hline$>20$ & 15 & 0.66 & 0.47 to 0.93 & 22.63 & 4 & 0.0002 \\
\hline \multicolumn{7}{|c|}{$\begin{array}{l}\text { Frequency of smoking in } \\
\text { room with child }\end{array}$} \\
\hline Never & 44 & 1.00 & - & & & \\
\hline Occasionally & 118 & 0.76 & 0.64 to 0.91 & & & \\
\hline Frequently & 48 & 0.60 & 0.48 to 0.75 & & & \\
\hline Every day & 228 & 0.66 & 0.56 to 0.77 & 28.86 & 3 & $<0.000$ \\
\hline \multicolumn{7}{|c|}{$\begin{array}{l}\text { No of parents who } \\
\text { smoke }\end{array}$} \\
\hline Father only & 66 & 1.00 & - & & & \\
\hline Mother only & 194 & 0.78 & 0.67 to 0.92 & & & \\
\hline Both parents & 178 & 0.79 & 0.68 to 0.92 & 10.73 & 2 & 0.005 \\
\hline
\end{tabular}

$\mathrm{IRR}=$ the incidence rate ratio, the frequency of the number of contacts as a ratio of the frequency in the reference category (the first category of each factor).

Table 2 Influence of non-smoking factors on frequency of asthma contacts: univariate analysis

\begin{tabular}{|c|c|c|c|c|c|c|}
\hline Factor & $n$ & $I R R$ & $C I(95 \%)$ & Model $\chi^{2}$ & $d f$ & $p$ value \\
\hline \multicolumn{7}{|l|}{ BTS step } \\
\hline 1 & 83 & 1.00 & - & & & \\
\hline 2 & 49 & 1.70 & 1.35 to 2.16 & & & \\
\hline 3 & 272 & 1.88 & 1.58 to 2.25 & & & \\
\hline $4 \& 5$ & 34 & 2.77 & 2.21 to 3.48 & 86.88 & 3 & $<0.0001$ \\
\hline \multicolumn{7}{|c|}{ Age of child (years) } \\
\hline $2-3$ & 49 & 1.00 & - & & & \\
\hline 4 & 44 & 0.74 & 0.06 to 0.90 & & & \\
\hline $5-7$ & 147 & 0.62 & 0.53 to 0.73 & & & \\
\hline $8-10$ & 129 & 0.51 & 0.43 to 0.60 & & & \\
\hline $11-12$ & 34 & 0.40 & 0.32 to 0.49 & 95.51 & 4 & $<0.0001$ \\
\hline \multicolumn{7}{|c|}{$\begin{array}{l}\text { Perceived severity of } \\
\text { asthma }\end{array}$} \\
\hline Very mild & 96 & 1.00 & - & & & \\
\hline Mild & 181 & 1.13 & 0.96 to 1.32 & & & \\
\hline Moderate & 128 & 1.61 & 1.37 to 1.89 & & & \\
\hline Severe & 28 & 1.66 & 1.32 to 2.10 & & & \\
\hline Very severe & 6 & 3.66 & 2.69 to 4.98 & 90.91 & 4 & $<0.0001$ \\
\hline \multicolumn{7}{|c|}{ Age of index parent (years) } \\
\hline$<25$ & 33 & 1.00 & - & & & \\
\hline $26-30$ & 126 & 0.80 & 0.65 to 0.97 & & & \\
\hline $31-35$ & 148 & 0.71 & 0.58 to 0.86 & & & \\
\hline $36-40$ & 80 & 0.67 & 0.54 to 0.84 & & & \\
\hline \multirow{2}{*}{\multicolumn{7}{|c|}{ Sex of child }} \\
\hline & & & & & & \\
\hline Male & 290 & 1.00 & - & & & \\
\hline Female & 148 & 1.12 & 1.00 to 1.25 & 3.78 & 1 & 0.05 \\
\hline \multicolumn{7}{|c|}{ No of children in family } \\
\hline 1 & 92 & 1.00 & - & & & \\
\hline 2 & 208 & 1.03 & 0.89 to 1.18 & & & \\
\hline 3 & 92 & 0.94 & 0.80 to 1.12 & & & \\
\hline 4 & 46 & 0.75 & 0.60 to 0.94 & 10.39 & 3 & 0.02 \\
\hline
\end{tabular}

IRR = the incidence rate ratio, the frequency of the number of contacts as a ratio of the frequency in the reference category (the first category of each factor).
DATA ANALYSIS

SPSS was used to calculate frequencies and construct tables. Poisson regression models were then fitted using Stata release 5. Models were obtained using a forward selection method. Categorical variables with $\mathrm{k}$ levels were fitted with $\mathrm{k}-1$ dummy variables, the lowest level being used as the comparator. The entry level set for inclusion in the model was $\mathrm{p}<0.05$.

\section{Results}

The mean age of the children was 7.1 years (range 2-12) and 290 (66\%) were boys. The mean age of the parents was 33 years (range $19-53)$ and $77 \%(n=335)$ were married or lived with a partner. The majority $(62 \%)$ of families lived in rented accommodation. The head of the household in 228 of the families $(52 \%)$ was in manual employment, $124(28 \%)$ were in non-manual employment, and in the remaining 86 families the head of the household was not employed. Most of the children $(62 \%)$ were maintained on low dose inhaled corticosteroids (step 3 of the BTS guidelines), ${ }^{8}$ $11 \%$ on sodium cromoglycate (step 2 ), $8 \%$ on high dose inhaled corticosteroids (steps 4 and 5), and $19 \%$ were prescribed $\beta_{2}$ agonists alone (step 1). Salivary cotinine levels ranged from a non-detectable level to $21.2 \mathrm{ng} / \mathrm{ml}$.

The children made a median number of two contacts for asthma during the 12 month period (range $0-20)$. Most of the children (77\%) had 1-6 contacts, $10 \%$ had $>6$, and $14 \%$ had none. The mean number of non-respiratory visits was two (range $0-12$ ); $20 \%$ of children had no consultations for non-respiratory events, $74 \%$ had $1-6$ contacts, and $6 \%$ had $>6$.

INFLUENCE OF SMOKING FACTORS ON FREQUENCY OF CONTACTS FOR ASTHMA Several factors had significant associations with the frequency of contacts for asthma (table 1). The findings are expressed in terms of the incidence rate ratio (IRR) with the reference category as the first category of each factor. An inconsistent relationship was found between salivary cotinine levels in the children and health service contacts for asthma: those with high cotinine levels had an increased contact rate (IRR=1.19) whereas children with moderate cotinine levels had a reduced likelihood of consultations for asthma (IRR=0.91). Higher levels of smoking in the home and in the presence of the child by the index parent resulted in substantially fewer contacts for asthma. The frequency of health care visits for asthma also fell with the number of parents who smoked, the largest fall occurring when the mother smoked.

Several factors unrelated to smoking also had a statistically significant relationship with asthma contacts (table 2). Severity of asthma as defined by BTS step showed a linear trend between increased contacts and increasing BTS step. Similarly, the number of contacts with health care services increased sequentially when parents perceived the child's asthma to be severe. Younger children and children of young parents were more likely to have contacts with health care services. Girls had more contacts than boys and belonging to a 
Table 3 Asthma contacts: factors exerting statistically significant independent effects

\begin{tabular}{|c|c|c|c|c|}
\hline Signifiicant factors & $n$ & $I R R$ & $95 \% C I$ & $p$ value \\
\hline \multicolumn{5}{|l|}{ Age of child (years) } \\
\hline $2-3$ & 49 & 1.00 & - & \\
\hline 4 & 44 & 0.77 & 0.63 to 0.94 & 0.012 \\
\hline $5-7$ & 147 & 0.64 & 0.54 to 0.75 & $<0.0001$ \\
\hline $8-10$ & 129 & 0.49 & 0.41 to 0.59 & $<0.0001$ \\
\hline $11-12$ & 69 & 0.41 & 0.33 to 0.51 & $<0.0001$ \\
\hline \multicolumn{5}{|c|}{ Perceived severity of asthma } \\
\hline Very mild & 95 & 1.00 & - & - \\
\hline Mild & 181 & 1.08 & 0.92 to 1.27 & 0.36 \\
\hline Moderate & 128 & 1.34 & 1.13 to 1.58 & 0.001 \\
\hline Severe & 28 & 1.49 & 1.17 to 1.89 & 0.001 \\
\hline Very severe & 6 & 2.68 & 1.94 to 3.70 & $<0.0001$ \\
\hline \multicolumn{5}{|c|}{ Severity of asthma by BTS step } \\
\hline Step 1 & 83 & 1.00 & - & - \\
\hline Step 2 & 49 & 1.94 & 1.52 to 2.46 & $<0.0001$ \\
\hline Step 3 & 272 & 2.01 & 1.67 to 2.42 & $<0.0001$ \\
\hline Step $4 \& 5$ & 34 & 2.40 & 1.89 to 3.06 & $<0.0001$ \\
\hline \multicolumn{5}{|c|}{ No of parents who smoke } \\
\hline Father only & 66 & 1.00 & - & - \\
\hline Mother only & 194 & 0.76 & 0.64 to 0.89 & 0.001 \\
\hline Both parents & 178 & 0.78 & 0.66 to 0.93 & 0.006 \\
\hline \multicolumn{5}{|c|}{ No of children in family } \\
\hline 1 & 92 & 1.00 & - & - \\
\hline 2 & 208 & 0.99 & 0.85 to 1.14 & 0.86 \\
\hline 3 & 92 & 0.84 & 0.71 to 1.00 & 0.05 \\
\hline 4 or more & 46 & 0.74 & 0.59 to 0.93 & 0.01 \\
\hline \multicolumn{5}{|l|}{ Child cotinine level } \\
\hline Low & 135 & 1.00 & - & - \\
\hline Medium & 153 & 0.95 & 0.82 to 1.11 & 0.52 \\
\hline High & 150 & 1.15 & 0.98 to 1.34 & 0.08 \\
\hline
\end{tabular}

$\mathrm{IRR}=$ the incidence rate ratio, the frequency of the number of contacts as a ratio of the frequency in the reference category (the first category of each factor).

Table 4 Influence of smoking factors on frequency of non-respiratory contacts: univariate analysis

\begin{tabular}{|c|c|c|c|c|c|c|}
\hline Factor & $n$ & $I R R$ & $95 \% C I$ & Model $\chi^{2}$ & $d f$ & $p$ value \\
\hline \multicolumn{7}{|c|}{ Child cotinine level } \\
\hline Low & 135 & 1.00 & - & & & \\
\hline Medium & 153 & 0.98 & 0.84 to 1.14 & & & \\
\hline High & 150 & 1.15 & 0.99 to 1.34 & 5.49 & 2 & 0.06 \\
\hline \multicolumn{7}{|c|}{$\begin{array}{l}\text { Amount smoked by } \\
\text { index parent } \\
\text { (cigarettes/day) }\end{array}$} \\
\hline$<10$ & 62 & 1.00 & - & & & \\
\hline $10-19$ & 236 & 1.03 & 0.86 to 1.24 & & & \\
\hline $20-29$ & 116 & 0.94 & 0.76 to 1.15 & & & \\
\hline$>30$ & 24 & 0.99 & 0.72 to 1.35 & 1.61 & 3 & 0.66 \\
\hline \multicolumn{7}{|c|}{$\begin{array}{l}\text { Amount smoked in the } \\
\text { home by index } \\
\text { parent } \\
\text { (cigarettes/day) }\end{array}$} \\
\hline $0-5$ & 104 & 1.00 & - & & & \\
\hline $6-10$ & 191 & 1.00 & 0.85 to 1.16 & & & \\
\hline $11-15$ & 78 & 0.86 & 0.70 to 1.04 & & & \\
\hline $16-20$ & 50 & 0.96 & 0.76 to 1.19 & & & \\
\hline$>20$ & 15 & 1.35 & 0.99 to 1.84 & 7.79 & 4 & 0.10 \\
\hline \multicolumn{7}{|c|}{$\begin{array}{l}\text { Frequency of smoking } \\
\text { in room with child }\end{array}$} \\
\hline Never & 44 & 1.00 & - & & & \\
\hline Occasionally & 118 & 0.74 & 0.60 to 0.93 & & & \\
\hline Frequently & 48 & 0.78 & 0.60 to 1.02 & & & \\
\hline Every day & 228 & 0.92 & 0.75 to 1.12 & 10.61 & 3 & 0.01 \\
\hline \multicolumn{7}{|c|}{$\begin{array}{l}\text { No of parents who } \\
\text { smoke }\end{array}$} \\
\hline Father only & 66 & 1.00 & - & & & \\
\hline Mother only & 194 & 1.25 & 1.03 to 1.50 & & & \\
\hline Both parents & 178 & 1.02 & 0.84 to 1.24 & 10.94 & 2 & 0.004 \\
\hline
\end{tabular}

IRR $=$ the incidence rate ratio, the frequency of the number of contacts as a ratio of the frequency in the reference category (the first category of each factor).

large family was associated with fewer visits. There was no significant association between contacts for asthma and social class or housing tenure.

ASTHMA CONTACTS: MULTIVARIATE MODEL The factors which showed significant effects in the univariate analysis were fitted in a Poisson multiple regression model to see which exerted independent effects. The age of the child continued to have a highly statistically significant association (table 3). Clear linear relationships were found between the number of contacts and the severity of asthma defined by BTS step, parental perception of asthma severity, and family size. Smoking by the mother also exerted a significant independent effect. Cotinine levels of the children were significantly associated with asthma contacts but, as in the univariate analysis, the effect was weak and inconsistent. Although a high cotinine level was associated with an increase in asthma contacts (IRR=1.15), those with moderate cotinine levels made fewer visits than children with low cotinine levels $(\mathrm{IRR}=0.95)$.

NON-RESPIRATORY CONTACTS

To assess the specificity of the association between the smoking factors and asthma visits to health care services, the factors influencing non-respiratory visits were investigated. The pattern of association between cotinine levels of children and non-respiratory contacts was similar to that for asthma visits, but failed to reach statistical significance (table 4). Other smoking factors showed a much weaker and inconsistent relationship with the frequency of visits for non-respiratory causes. Only two achieved statistical significance: for smoking in the same room the pattern was U-shaped rather than linear, and for number of parents smoking the effect was in the opposite direction to that seen for asthma contacts.

\section{Discussion}

The main aim of this study was to determine whether passive smoking, measured by salivary cotinine levels, was associated with asthma morbidity, as measured by health service contacts. We have found a complex relationship where, compared with low exposure, intermediate exposure was associated with reduced morbidity and high exposure resulted in increased morbidity. This pattern was maintained after adjustment for potential confounding variables. The lack of a clear relationship was unexpected, particularly since an earlier study showed a clear dose-response relationship between respiratory function and cotinine levels. ${ }^{10}$

Several measures of parental smoking (amount smoked at home, frequency of smoking in front of the child, and number of parents who smoke) also influenced the number of contacts. However, this was not in the direction expected: as the level of smoking increased the number of visits for asthma decreased. At the home with the highest reported smoking level there was a $34 \%$ reduction in visit frequency. What makes this finding more compelling is that the association between parental smoking and non-respiratory contacts is very much weaker and the direction of effect inconsistent. This finding supports the idea that the relationship between smoking levels and asthma contacts is a real phenomenon and not the result of confounding.

One explanation for the association between smoking and asthma could be that heavy smoking reduces the awareness of parents to the children's asthma symptoms. Heavy smokers may be less concerned about respiratory 
symptoms associated with smoking. A second possibility is that the heavy smokers are more reluctant to take their children to the doctor because they do not wish to be told to stop smoking. ${ }^{11}$ Parents who suspect that their smoking could affect their child's asthma may be less willing to receive anti-smoking advice and could seek to avoid it. Whichever explanation is correct, action is required to ensure that asthmatic children with parents who are heavy smokers receive adequate medical attention.

A second important finding from this study is the existence of several other powerful factors which influence visit frequency. Thus, there is a strong effect of age on all visits, with younger children having many more visits. The number of children in the family also affects the number of visits for asthma. Larger family size has previously been reported to be associated with increased consultation rates. ${ }^{12}$ This could reflect increased parental experience in dealing with illness, but equally could be due to the difficulty of visiting the GP with several small children.

Perceived severity of asthma and actual severity, as measured by BTS treatment step, were also strongly and independently associated with asthma visits. These factors had a weak and inconsistent effect on nonrespiratory contacts. Concerns about disease or symptom severity are recognised to exert a powerful influence on consultation rates. ${ }^{12-14} \mathrm{It}$ is worth noting that parent perception of severity is as important as actual severity; in the present study perceived severity exerted a powerful effect independent of actual severity as measured by treatment step for asthma contacts.

The finding that non-clinical factors are powerful determinants of health care contacts has an important consequence. Health care contacts are often used as a proxy for morbidity in studies of asthma and have been used, as in the present study, to investigate the impact of passive smoking on asthma. ${ }^{15}{ }^{16}$ As non-clinical factors are the dominant influence on contact rates, potentially misleading findings could arise if they are not taken into account. Health care utilisation should be recognised as a poor measure of asthma morbidity.

This study found that social factors were unrelated to numbers of visits. The study sample was weighted towards manual workers, reflecting the social class distribution of smoking. However, there were sufficient numbers of non-manual workers $(n=86,20 \%)$ to detect even moderate effects had these existed. This finding may appear unexpected, but it is supported by several studies of consultations by children. ${ }^{12-14} 17$ The lack of a role for social factors on visits for medical conditions strengthens the theory that it is personal psychological factors and family circumstance which determine visit patterns.

There is clear evidence that passive smoking increases the prevalence of asthma. ${ }^{3}$ However, the studies on severity and frequency of asthma attacks give conflicting findings; although most support an association between passive smoking and disease severity, some have found an inverse relationship. ${ }^{4}$ Furthermore, few of these studies adjusted for potential confounding factors. This could be a serious omission since the present study has found that several powerful factors influence the frequency of health service contacts.

This study has failed to resolve the issue of whether passive smoking exacerbates asthma. High levels of passive smoking may cause increased morbidity as measured by health care contacts. However, non-clinical factors have a dominant influence on visit frequency, making number of visits a poor measure of asthma morbidity. This finding casts doubt on all studies which have used this proxy measure for morbidity but have not controlled for nonclinical factors.

The main finding is serendipitous; increased parental smoking in the home is associated with a reduction in health care contacts for asthma. This could be due to a lack of awareness about asthma symptoms among heavy smokers, or heavy smokers may be reluctant to visit their doctor. The finding suggests that children with asthma who have parents who smoke may not be receiving adequate management.

The collection of the questionnaire data and the salivary cotinine levels was supported by a grant from the Wellcome Trust $(039282 / \mathrm{Z} / 93 / \mathrm{Z})$. Review of the GP case notes and the subsequent analysis were supported by a grant from the Scottish Executive Chief Scientist Office (K/OPR/2/2/D328). We are grateful to the general practitioners and their staff from the practices in Tayside and Fife who assisted in the study.

Conflict of interest: none.

1 Strachan DP, Cook DG. Parental smoking and lower respiratory illness in infancy and early childhood. Thorax 1997;52:905-14.

2 Strachan DP. Parental smoking, middle ear disease and adenotonsillectomy in children. Thorax 1998;53:50-6.

3 Cook DG, Strachan DP. Parental smoking and prevalence of respiratory symptoms and asthma in school aged children. Thorax 1997;52:1081-94.

4 Strachan DP. Parental smoking and childhood asthma: longitudinal and case-control studies. Thorax 1998;53:20412 .

5 Irvine L, Crombie IK, Clark RA, et al. What determines levels of passive smoking in children with asthma? Thorax 1997;52:766-9.

6 Irvine L, Crombie IK, Clark RA, et al. Advising parents of asthmatic children on passive smoking: randomised controlled trial. BMF 1999;318:1456-9.

7 Office of Population Censuses and Surveys. Standard occupational classification. London: HMSO, 1990.

8 British Thoracic Society. Guidelines on the management of asthma. Thorax 1993;48:S1-24.

9 Feyerabend C, Russell MAH. A rapid gas-liquid chromatographic method for the determination of cotinine and nicotine in biological fluids. F Pharm Pharmacol 1990;42:450-2.

10 Cook DG, Whincup PH, Papacosta O, et al. Relation of passive smoking as assessed by salivary cotinine concentration and questionnaire to spirometric indices in children. Thorax 1993;48:14-20

11 Butler CC, Pill R, Stott NCH. Qualitative study of patients' perceptions of doctors' advice to quit smoking: implica-
tions for opportunistic health promotion. BMF 1998;316: 1878-81.

12 Duncan JK, Taylor RJ, Fordyce ID. Factors associated with variation in the consultation rates of children aged under five years. $\mathcal{F}$ Coll Gen Pract 1987;37:251-4.

13 Wyke S, Hewison J, Russell IT. Respiratory illness in children: what makes parents decide to consult? $\mathrm{Br} \mathcal{F} \mathrm{Gen}$ Pract 1990;40:226-9.

14 Osman LM, Dunt D. Factors influencing mothers' decisions to consult a general practitioner about their children's illnesses. Br F Gen Pract 1995;45:310-2.

15 Evans D, Levison MJ, Feldman CH, et al. The impact of passive smoking on emergency room visits of urban
children with asthma. Am Rev Respir Dis 1987;135:567-72.

16 Chilmonczyk BA, Salmun LM, Megathlin KN, et al. Association between exposure to environmental tobacco smoke and exacerbations of asthma in children. $N$ Engl $\mathcal{f} \mathrm{Med}$ 1993;328:1665-9.

17 Baker D, Taylor H, Asthma Survey Team of the Avon Longitudinal Study of Pregnancy and Childhood. Inequality in health and health service use for mothers of young children in south west England. $\mathcal{F}$ Epidemiol Community Health in south west 